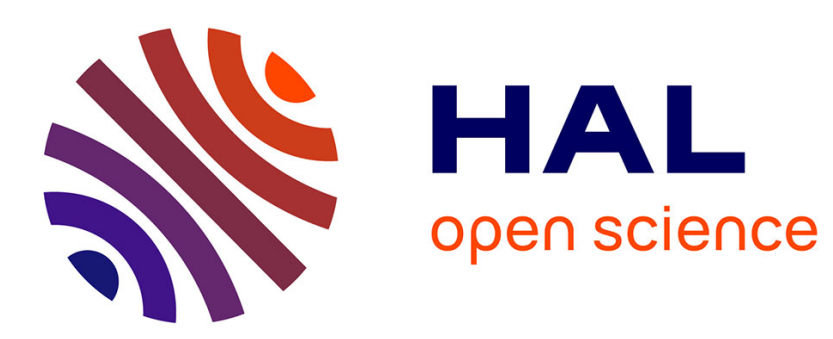

\title{
Le polyimide-alumine: un isolant performant utilisé dans un aimant supraconducteur refroidi à l'hélium superfluide
}

\author{
P. Libeyre
}

\section{- To cite this version:}

P. Libeyre. Le polyimide-alumine: un isolant performant utilisé dans un aimant supraconducteur refroidi à l'hélium superfluide. Revue de Physique Appliquée, 1988, 23 (11), pp.1761-1765. 10.1051/rphysap:0198800230110176100 . jpa-00246005

\section{HAL Id: jpa-00246005 https://hal.science/jpa-00246005}

Submitted on 1 Jan 1988

HAL is a multi-disciplinary open access archive for the deposit and dissemination of scientific research documents, whether they are published or not. The documents may come from teaching and research institutions in France or abroad, or from public or private research centers.
L'archive ouverte pluridisciplinaire HAL, est destinée au dépôt et à la diffusion de documents scientifiques de niveau recherche, publiés ou non, émanant des établissements d'enseignement et de recherche français ou étrangers, des laboratoires publics ou privés. 


\title{
Le polyimide-alumine : un isolant performant utilisé dans un aimant supraconducteur refroidi à l'hélium superfluide
}

\author{
P. Libeyre \\ Association EURATOM-CEA sur la Fusion Contrôlée, DRFC/STIF, Centre d'Etudes Nucléaires de \\ Cadarache, 13108 Saint-Paul Lez Durance Cedex, France
}

(Reçu le 11 avril 1988, accepté le 13 juillet 1988)

\begin{abstract}
Résumé. - L'aimant toroïdal de TORE SUPRA se compose de 18 bobines, chacune d'entre elles étant constituée d'un bobinage supraconducteur enfermé dans un boîtier épais en acier inoxydable destiné à supporter les efforts électromagnétiques. Le bobinage supraconducteur est refroidi par un bain d'hélium superfluide à $1,8 \mathrm{~K}$; le boîtier épais est refroidi par une circulation d'hélium supercritique à $4,2 \mathrm{~K}$. Le système de calage entre bobinage et boîtier épais assure à la fois l'isolation thermique entre $1,8 \mathrm{~K}$ et $4,2 \mathrm{~K}$ et la transmission des efforts du bobinage au boîtier. Pour répondre à ces deux impératifs il s'est avéré nécessaire de développer un matériau nouveau : le polyimide-alumine. Le programme de développement a abouti à la mise au point d'une production industrielle de ce matériau, ce qui a permis la réalisation de l'aimant conformément aux spécifications.
\end{abstract}

\begin{abstract}
The toroidal magnet of TORE SUPRA is made up of 18 coils, each one consisting of a superconducting winding enclosed inside a thick stainless steel casing dedicated to withstand the electromagnetic forces. The superconducting winding is cooled by a superfluid helium bath at $1.8 \mathrm{~K}$; the thick casing is cooled by a supercritical helium flow at $4.2 \mathrm{~K}$. The chocks between winding and thick casing provide as well the thermal insulation between $1.8 \mathrm{~K}$ and $4.2 \mathrm{~K}$ as the transmission of loads from the winding to the casing. To meet this two requirements it prooved out necessary to develop a new material : polyimide-alumina. The development program resulted in the achievement of an industrial production of this material, which allowed the realisation of the magnet in conformity with the specifications.
\end{abstract}

\section{L'aimant toroïdal de TORE SUPRA.}

Dans le cadre des recherches sur la Fusion contrôlée, l'Association EURATOM-CEA est engagée dans la réalisation d'un dispositif tokamak appelé TORE SUPRA. Cet appareil comporte un aimant toroïdal composé de 18 bobines supraconductrices dont le bobinage est refroidi à $1,8 \mathrm{~K}$ par un bain d'hélium superfluide.

1.1 CARACTÉRISTIQues GÉNÉRALES. - Les principaux paramètres du tokamak sont les suivants :

$\begin{array}{lll}\text { Grand rayon du plasma } & 2,25 \mathrm{~m} \\ \text { Petit rayon du plasma } & 0,70 \mathrm{~m} \\ \text { Induction magnétique sur l'axe } & 4,5 & \mathrm{~T} \\ \text { Induction magnétique maximum } & 9 & \mathrm{~T}\end{array}$

Les caractéristiques de l'aimant toroïdal sont :
Grand rayon
$2,443 \mathrm{~m}$
Rayon intérieur d'une bobine

\begin{abstract}
Rayon extérieur d'une bobine $\quad 1,485 \mathrm{~m}$
Courant dans le conducteur $1400 \mathrm{~A}$

Solénation du bobinage $\quad 2,84$ MA
\end{abstract}

1.2 Principaux Choix technologiques. - Le supraconducteur est un alliage de niobium-titane. Le fil supraconducteur se présente sous forme d'un composite monolithe constitué d'une matrice de cuivre dans laquelle sont noyés les filaments de $\mathrm{NbTi}$ (dimensions de la section du fil : $2,8 \mathrm{~mm} \times 5,6 \mathrm{~mm}$ ).

Le refroidissement est assuré par un bain d'hélium superfluide à $1,8 \mathrm{~K}$ pressurisé à $0,1 \mathrm{MPa}$.

Seul le bobinage lui-même est plongé dans le bain d'hélium superfluide ainsi que le montre la figure 1.

Il existe donc 3 niveaux de refroidissement :

- le bobinage à $1,8 \mathrm{~K}$;

- son boîtier épais à $4,2 \mathrm{~K}$;

- les écrans thermiques qui entourent les bobines à $77 \mathrm{~K}$. 


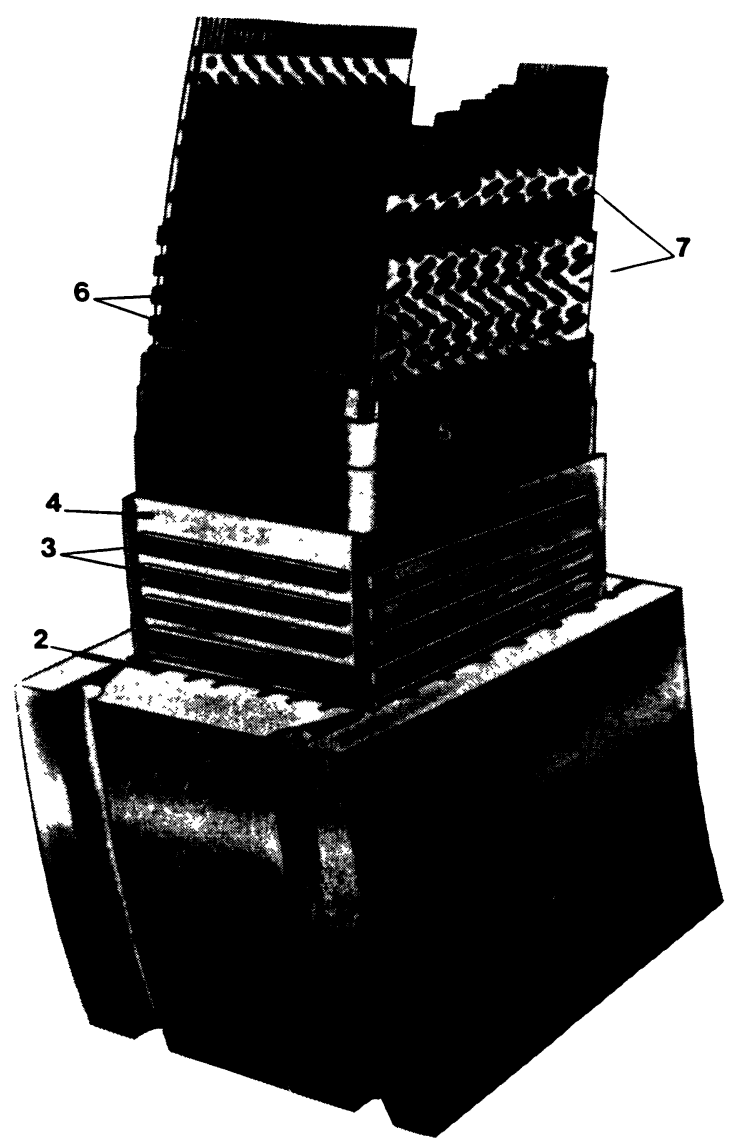

1. Boittier épais

2. Canaux $4,2 \mathrm{~K}$

3. Barrettes de calage polyimide-alumine

4. Enceinte $1,8 \mathrm{~K}$ (boîtier mince)

5. Isolation masse

6. Barrettes de calage

7. Intercalaires

8. supraconducteur

Fig. 1. - Structure d'une bobine supraconductrice de l'aimant toroïdal.

[Structure of a superconducting coil of the toroidal magnet.]

L'isolation thermique entre l'aimant et les écrans thermiques est réalisée par le vide, l'ensemble étant enfermé dans un cryostat torique. Il n'est pas nécessaire à ce niveau de disposer de structure de calage car les écrans supportent eux-mêmes les efforts auxquels ils sont soumis. Par contre les efforts électromagnétiques qui apparaissent dans le bobinage doivent être transférés vers le boîtier épais qui l'entoure. Il est donc nécessaire de disposer d'une isolation thermique entre le bobinage et le

Tableau I. - Comparaison des différents isolants.

[Comparison between different insulators.]

\begin{tabular}{|c|c|c|c|c|c|}
\hline \multirow[b]{2}{*}{ Propriétés } & \multirow[t]{2}{*}{ Epoxides } & \multicolumn{2}{|c|}{ Polyamides } & \multicolumn{2}{|c|}{ Polyimides } \\
\hline & & Nylon & Aramide & Vespel & Kerimid \\
\hline $\begin{array}{r}300 \mathrm{~K} \\
R m(\mathrm{MPa}) \\
4,2 \mathrm{~K}\end{array}$ & $100-170$ & $50-90$ & 380 & 370 & $>590$ \\
\hline $\begin{array}{c}\lambda\left(\mathrm{W} \cdot \mathrm{m}^{-1} \cdot \mathrm{K}^{-1}\right) \\
4,2 \mathrm{~K}\end{array}$ & $5-7 \times 10^{-2}$ & $1,25 \times 10^{-2}$ & $3,5 \times 10^{-2}$ & $1,1 \times 10^{-2}$ & $3,75 \times 10^{-2}$ \\
\hline $\begin{array}{l}\int_{77 \mathrm{~K}}^{300 \mathrm{~K}} \frac{\Delta \ell}{\ell} \\
\int_{4,2 \mathrm{~K}}^{300 \mathrm{~K}} \frac{\Delta \ell}{\ell}\end{array}$ & $10 \times 10^{-3}$ & $14,5 \times 10^{-3}$ & $6,5 \times 10^{-3}$ & $\begin{array}{l}7,7 \times 10^{-3} \\
9 \times 10^{-3}\end{array}$ & $7,4 \times 10^{-3}$ \\
\hline
\end{tabular}


boîtier qui soit capable de transmettre des efforts mécaniques importants.

1.3 CONDITIONS REQUISES POUR LES CALES D'ISOLATION THERMIQUE. - Le système de calage doit donc répondre à 3 impératifs :

- isolation thermique entre $1,8 \mathrm{~K}$ et $4,2 \mathrm{~K}$;

- résistance mécanique en compression élevée ;

- faible contraction thermique pour éviter l'apparition de jeux entre bobinage et boîtier.

On aboutit donc à 3 spécifications :

$$
\begin{gathered}
\lambda<10^{-2} \mathrm{~W} \cdot \mathrm{m}^{-1} \cdot \mathrm{K}^{-1} \text { à } 4,2 \mathrm{~K} \\
R m \geqq 500 \mathrm{MPa} \text { à } 4,2 \mathrm{~K} \\
\int_{4,2 \mathrm{~K}}^{300 \mathrm{~K}} \frac{\Delta \ell}{\ell} \leqq 4,5 \times 10^{-3} .
\end{gathered}
$$

\section{Programme de développement.}

Aucun matériau disponible sur le marché ne réunissant les 3 conditions spécifiées précédemment il s'est avéré nécessaire d'entreprendre un programme de développement en 1977 afin de mettre au point un nouveau matériau [1].

2.1 COMPARAISON DES DIVERS ISOLANTS DISPONIBLES. - La comparaison des principaux isolants disponibles est résumée dans le tableau I.

Le polyimide Kerimid de RHONE POULENC paraissait donc le plus intéressant.

Des considérations thermiques ayant montré qu'il serait intéressant de charger ces isolants pour en diminuer la conductivité thermique. On a envisagé deux types de charges couramment utilisées :

- les fibres de verre;

- la poudre d'alumine.

2.2 RÉSUlTATS DES ESSAIS. - Il est apparu que l'alumine permettait un meilleur abaissement de la conductivité thermique que les fibres de verre, aussi les essais ont porté sur la sélection de l'alumine et la composition du mélange. Les résultats ont été publiés en 1978 à la conférence ICMC de Munich. La figure 2 montre l'influence de la charge en poudre d'alumine sur la conductivité du polyimide. On constate un net abaissement de la conductivité thermique dans la gamme de température qui nous interresse.

La figure 3 indique pour 3 types d'alumine les différents résultats obtenus en faisant varier la composition du mélange.

On a indiqué pour chaque type d'alumine la surface spécifique et pour chaque échantillon la composition du mélange : par exemple les échantillons repérés $\mathrm{K} 35 \mathrm{~A}_{3} 65$ contenaient $35 \%$ de polyimide et $65 \%$ d'alumine.

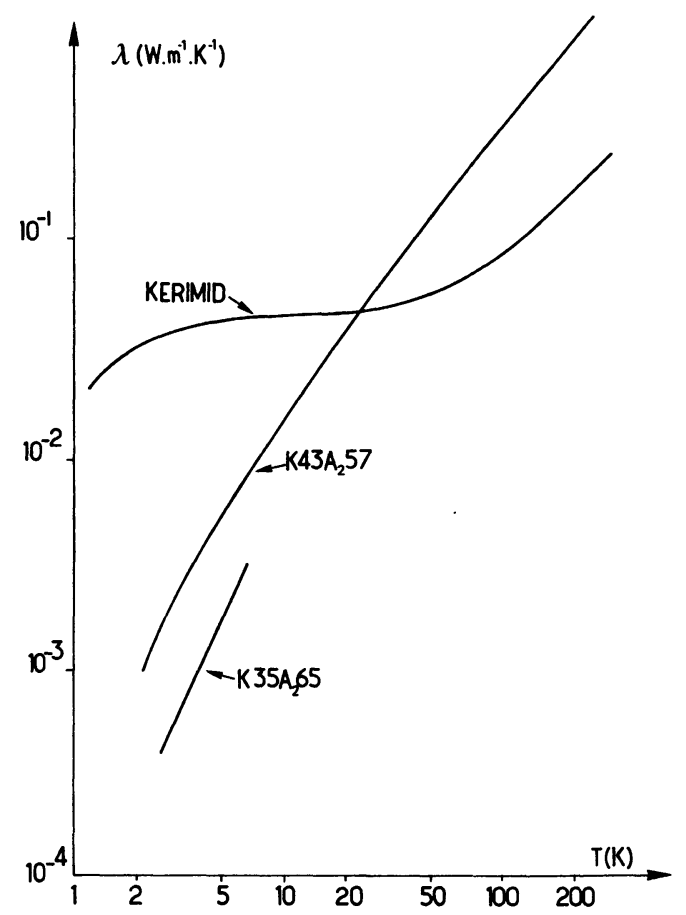

Fig. 2. - Influence de la charge en alumine sur la conductivité thermique.

[Influence of the alumina content on thermal conductivity.]

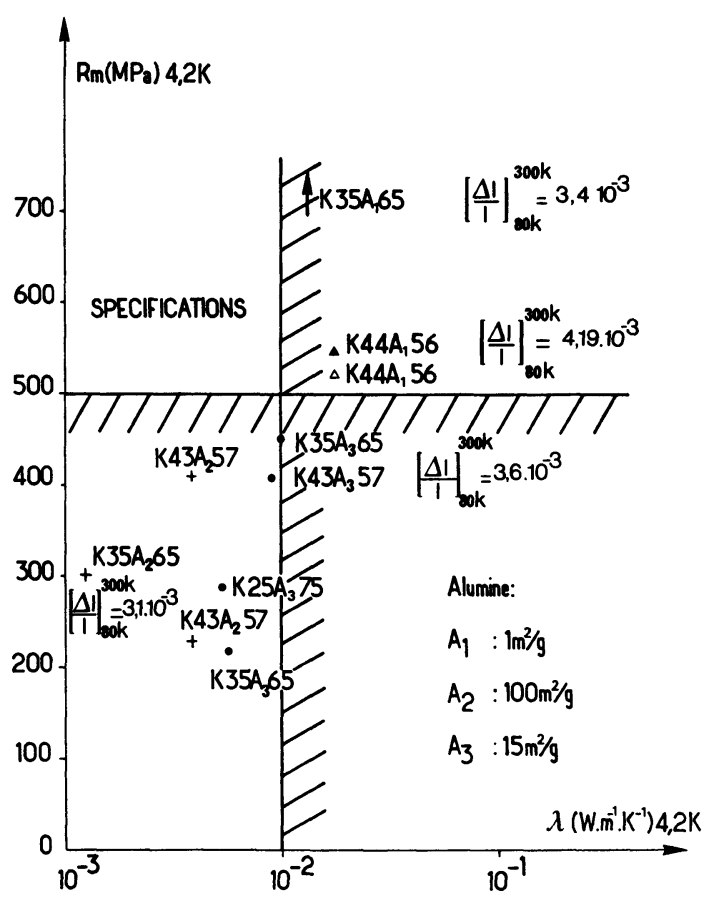

Fig. 3. - Comparaison des différents composites polyimide-alumine.

[Comparison between different polyimide-alumina compounds.]

Le tableau II résume les performances obtenues sur une série de 50 cales réalisée avec le composite K35 A 65 : 
Tableau II. - Caractéristique d'une présérie de cales en $\mathrm{K} 35 \mathrm{~A}_{3} 65$.

[Caracteristics of a preseries of chocks in K $35 A_{3} 65$.]

\begin{tabular}{|c|c|c|c|}
\hline Densité & $R m(300 \mathrm{~K})$ & $R m(4,2 \mathrm{~K})$ & {$\left[\frac{\Delta \ell}{\ell}\right]^{300 \mathrm{~K}}$} \\
\hline $1,91<d<2,15$ & $269 \mathrm{MPa}$ & $541 \mathrm{MPa}$ & $3,1 \times 10^{-3}$ \\
\hline
\end{tabular}

\section{Réalisation industrielle.}

3.1 MISE AU POINT DU PRODUIT. - Les essais précédents conduisirent à la réalisation industrielle de 10000 cales destinées à la réalisation d'une maquette de bobine supraconductrice essayée avec succès en 1980. Néanmoins le produit nécessitait une mise au point afin d'en faciliter la mise en œuvre industrielle et d'assurer une qualité fiable. Un contrat de développement du produit a donc été passé en 1982 avec RHONE-POULENC qui a abouti en 1983 à l'obtention d'un mélange sous forme de granulés, directement utilisable par moulage sous pression à chaud. Le coefficient de foisonnement du mélange qui était auparavant de 6 a ainsi pu être réduit à 2 .

La composition de la poudre à mouler est la suivante :

- polyimide Kerimid 1050 (RHONE POULENC) : $35 \%$;
- alumine A15Z (CRICERAM) + agent glissant démoulant : $64 \%$.

Les conditions de mise en œuvre sont les suivantes :

— température de moulage : $240-250{ }^{\circ} \mathrm{C}$;

— pression de moulage : $10-20 \mathrm{MPa}$;

- durée de cuisson : $2 \mathrm{~min} / \mathrm{mm}$;

— recuit : $16-24 \mathrm{~h}$ à $230-250^{\circ} \mathrm{C}$.

L'usinage s'effectue par découpage à la scie diamantée puis rectification.

3.2 CONTRÓlE DE LA FABRICATION. - Le contrôle de la fabrication s'est effectué de 2 manières :

- un contrôle systématique de chaque cale en lui faisant subir un essai de flexion;

- un contrôle par prélèvement d'échantillons sur lesquels ont été mesurés : la densité, la charge de rupture, la contraction thermique, la conductivité.

3.3 RÉSUltats obTENUS. - Une quantité de $3444 \mathrm{~kg}$ de poudre à mouler a été produite. Elle a permis la réalisation de 24507 cales par la société VIENNOT. Les propriétés mesurées sur échantillons sont récapitulées dans le tableau III.

\section{Conclusion.}

Le matériau développé a démontré son aptitude à la réalisation d'une production industrielle. Les cales ainsi obtenues ont servi à la réalisation de 20 bobines ( 1 bobine prototype +18 bobines de série +

Tableau III. - Propriétés du composite polyimide-alumine.

[Properties of the polyimide-aluminia compound.]

\begin{tabular}{|c|c|c|c|}
\hline Propriété & \multicolumn{2}{|c|}{ Conditions de mesures } & Valeur moyenne mesurée \\
\hline Densité & \multicolumn{2}{|c|}{$T=300 \mathrm{~K}$} & 2,24 \\
\hline \multirow[t]{2}{*}{ Charge de rupture } & compression & $\begin{array}{l}300 \mathrm{~K} \\
4,2 \mathrm{~K}\end{array}$ & $\begin{array}{l}471 \mathrm{MPa} \\
630 \mathrm{MPa}\end{array}$ \\
\hline & flexion & $300 \mathrm{~K}$ & $83 \mathrm{MPa}$ \\
\hline \multirow[t]{2}{*}{ Module d'Young } & compression & $\begin{array}{l}300 \mathrm{~K} \\
4,2 \mathrm{~K}\end{array}$ & $\begin{array}{l}15 \mathrm{GPa} \\
27 \mathrm{GPa}\end{array}$ \\
\hline & flexion & $300 \mathrm{~K}$ & $14 \mathrm{GPa}$ \\
\hline Contraction thermique & {$\left[\frac{\Delta \ell}{\ell}\right]_{4,2 \mathrm{~K}}^{300 \mathrm{~K}}$} & & $4,5 \times 10^{-3}$ \\
\hline Conductivité thermique & $1<T(\mathrm{~K})$ & $\begin{array}{l}4,2 \mathrm{~K} \\
1,8 \mathrm{~K}\end{array}$ & $\begin{array}{l}=7,03 \times 10^{-4} T^{1,58} \\
=0,68 \times 10^{-2} \mathrm{~W} \cdot \mathrm{m}^{-1} \cdot \mathrm{K}^{-1} \\
=0,18 \times 10^{-2} \mathrm{~W} \cdot \mathrm{m}^{-1} \cdot \mathrm{K}^{-1}\end{array}$ \\
\hline
\end{tabular}


1 bobine de réserve). Ces 20 bobines ont été testées achevée en 1987. La mise en service de TORE à basse température avec succès en 1985 et 1986. La SUPRA s'effectue au printemps de 1988 ; le premier construction de l'aimant toroïdal à Cadarache, s'est plasma a été obtenu le 6 avril 1988.

\section{Bibliographie}

[1] Claudet, G., Disdier, F. and Locatelli, M., Interesting low temperature thermal and mechanical properties of a particular powder-filled polyimide, ICMC (1978) Munich. 